\title{
Spectral and spatial shaping of a laser-produced ion beam for radiation-biology experiments
}

\author{
L. Pommarel, ${ }^{1}$ B. Vauzour, ${ }^{1, \dagger}$ F. Mégnin-Chanet, ${ }^{2,}$ E. Bayart, ${ }^{1}$ O. Delmas, ${ }^{1}$ F. Goudjil, ${ }^{3}$ \\ C. Nauraye ${ }^{3}$ V. Letellier, ${ }^{3}$ F. Pouzoulet, ${ }^{4}$ F. Schillaci, ${ }^{5}$ F. Romano, ${ }^{5}$ V. Scuderi, ${ }^{5}$ \\ G. A. P. Cirrone, ${ }^{5}$ E. Deutsch, ${ }^{2}$ A. Flacco, ${ }^{1,}{ }^{*}$ and V. Malka ${ }^{1}$ \\ ${ }^{1}$ Laboratoire d'Optique Appliquée, ENSTA ParisTech, CNRS, École Polytechnique, \\ Université Paris-Saclay, 828 bd des Maréchaux, 91762 Palaiseau Cedex, France \\ ${ }^{2}$ INSERM U1030, Gustave Roussy-Cancer Campus-Grand Paris, \\ 114 rue Edouard-Vaillant, 94805 Villejuif Cedex, France \\ ${ }^{3}$ Institut Curie-Centre de Protonthérapie d'Orsay, 91400 Orsay, France \\ ${ }^{4}$ Institut Curie, Translational Research Department, 15 rue Georges Clémenceau, 91898 Orsay Cedex, France \\ ${ }^{5}$ Istituto Nazionale di Fisica Nucleare, Laboratori Nazionali del Sud, Via S. Sofia 62, 95125 Catania, \\ Catania CT, Italy
}

(Received 10 October 2016; published 2 March 2017)

\begin{abstract}
The study of radiation biology on laser-based accelerators is most interesting due to the unique irradiation conditions they can produce, in terms of peak current and duration of the irradiation. In this paper we present the implementation of a beam transport system to transport and shape the proton beam generated by laser-target interaction for in vitro irradiation of biological samples. A set of four permanent magnet quadrupoles is used to transport and focus the beam, efficiently shaping the spectrum and providing a large and relatively uniform irradiation surface. Real time, absolutely calibrated, dosimetry is installed on the beam line, to enable shot-to-shot control of dose deposition in the irradiated volume. Preliminary results of cell sample irradiation are presented to validate the robustness of the full system.
\end{abstract}

DOI: 10.1103/PhysRevAccelBeams.20.032801

\section{INTRODUCTION}

Important progress on laser-driven ion acceleration by intense and ultrashort laser pulses has been achieved in the past decades. Repetition rates allow multiple laser shots per second, new proton energy records have been achieved [1], and new acceleration models have been proposed and are currently under investigation (radiation pressure acceleration [2], laser breakout afterburner [3], or collisionless shocks [4]). Available energies and beam repeatability open the possibility to study ionization effects caused by laserproduced proton beams on living tissues [5].

At present, the intrinsic properties of laser-accelerated beams are not suitable for direct irradiation of a sample, as they typically display a broad energy spectrum and a wide angular distribution [6]. A proper beam line needs to be set up in order to produce experimental conditions compatible with the needs of a biological study. Among other features,

\footnotetext{
*Corresponding author.

alessandro.flacco@polytechnique.edu

†Present address: CEA, DAM, DIF, F-91297 Arpajon, France.

†Present address: INSERM U1196/CNRS UMR9187, InstitutCurie Recherche, bâtiment 112, Centre Universitaire, 91405 Orsay Cedex, France.

Published by the American Physical Society under the terms of the Creative Commons Attribution 3.0 License. Further distribution of this work must maintain attribution to the author(s) and the published article's title, journal citation, and DOI.
}

a wide irradiation area of constant dose rate and the precise retrieval of the deposited dose are mandatory.

Ongoing research is dedicated to control charged particle beams. For instance, quadrupole doublets $[7,8]$ or triplets [9] and solenoids [10-12] were used to focus or transport them. In the context of laser-plasma accelerators, magnetic devices have been employed to focus and improve the stability of electron beams [13]. Ion beam characteristics modeling has been accomplished with either special target structures [14,15] or by using various types of magnetic apparatuses [16]. With the aim of performing radiobiological studies, a chicane made of four dipole magnets [17], and a quadrupole doublet [18] were also used, but none of them manages to meet all of the requirements for fruitful radiobiological studies, as listed by Kraft in [19].

Several research groups worldwide have been working to investigate the biological response to the high dose rates produced by laser-accelerated beams, even though strict requirements with respect to beam parameters such as particle energy, stability, dose monitoring devices and patient safety have to be fulfilled before a clinical application [20-22]. In many studies, the cell response was examined as a function of the laser-driven proton beam dose deposition that was adjusted either with the number of laser shots fired $[5,19]$, or by changing the incident beam energy in single-shot conditions [23]. Laser systems were optimized to accelerate electron beams suitable for cell irradiation experiments [24] and their effects were 
compared with that of continuous beams delivered by clinical linac [25]. Finally, efforts were also directed towards the development of dosimetric systems $[23,26]$.

In the present experiment, a system of four permanent magnet quadrupoles (PMQ) is used to transport a chosen spectral component of the proton beam far from its generation point $(1 \mathrm{~m})$. The intrinsic energy selection allows one to obtain a wide irradiation surface $(\varnothing 2 \mathrm{~cm})$ with a relatively uniform dose deposition for in vitro studies. Absolutely calibrated dosimetry was established and confirmed by the survival rate of the cell lineage HCT116, indicating a deposited dose of $1.15 \pm 0.25 \mathrm{~Gy} /$ shot.

\section{PROTON SOURCE}

The experiments have been conducted on the SAPHIR laser facility at the Laboratoire d'Optique Appliquée. The proton source is provided by a $200 \mathrm{TW}$ Ti:sapphire laser, which was focused on a $5 \mu \mathrm{m}$ thick titanium foil with a $45^{\circ}$ incidence angle by a $30^{\circ}$ off-axis parabolic mirror, having an $f$-number of $f / 3.4$. The laser delivers $3 \mathrm{~J}$ per pulse on target, with a pulse duration of $25 \mathrm{fs}$ and a high temporal contrast $\left(10^{-10}\right)$. The focal spot diameter was $10 \mu \mathrm{m}$ at $1 / e^{2}$, providing a peak intensity of $\mathrm{I}_{0}=4 \times 10^{19} \mathrm{~W} \mathrm{~cm}^{-2}$ at $\lambda=800 \mathrm{~nm}$. The target was chosen to be thick enough to limit the detrimental effects of the laser temporal contrast on the spatial and energy stability of the accelerated proton beam [27].

The protons were generated by target normal sheath acceleration (TNSA) [28,29]: laser-heated electrons drive the expansion of the thin metal foil, creating a strong transient accelerating field $\left(\sim \mathrm{TV} \mathrm{m}^{-1}\right)$ which in turn extracts, along the normal direction of the target rear surface, protons and carbon ions coming from water and organic contaminants. TNSA proton beams are characterized by a large divergence angle and energy spread (Boltzmann energy distribution with a cutoff), these features depending on the laser and target parameters [6]. In our experimental conditions, the beam had a cutoff energy of $8 \mathrm{MeV}$ and a FWHM average divergence angle of $24^{\circ}$ at $5 \mathrm{MeV}$. In Fig. 1 is shown the beam divergence for several energies, obtained from a six-layer stack of Gafchromic HD-V2 radiochromic films recording the beam spatial profile averaged over 25 shots $4 \mathrm{~cm}$ behind the interaction point.

The large angular divergence and energy spectrum, but also the presence of other types of ionizing radiations ( $x$ rays, electrons, heavy ions such as carbon and oxygen in various ionization states) makes nonrealistic the direct use of the beam for any practical application.

\section{PROTON BEAM TRANSPORT AND SHAPING}

\section{A. Transport}

A set of four permanent magnet quadrupoles designed by INFN-LNS researchers [30-32] was used for this purpose.
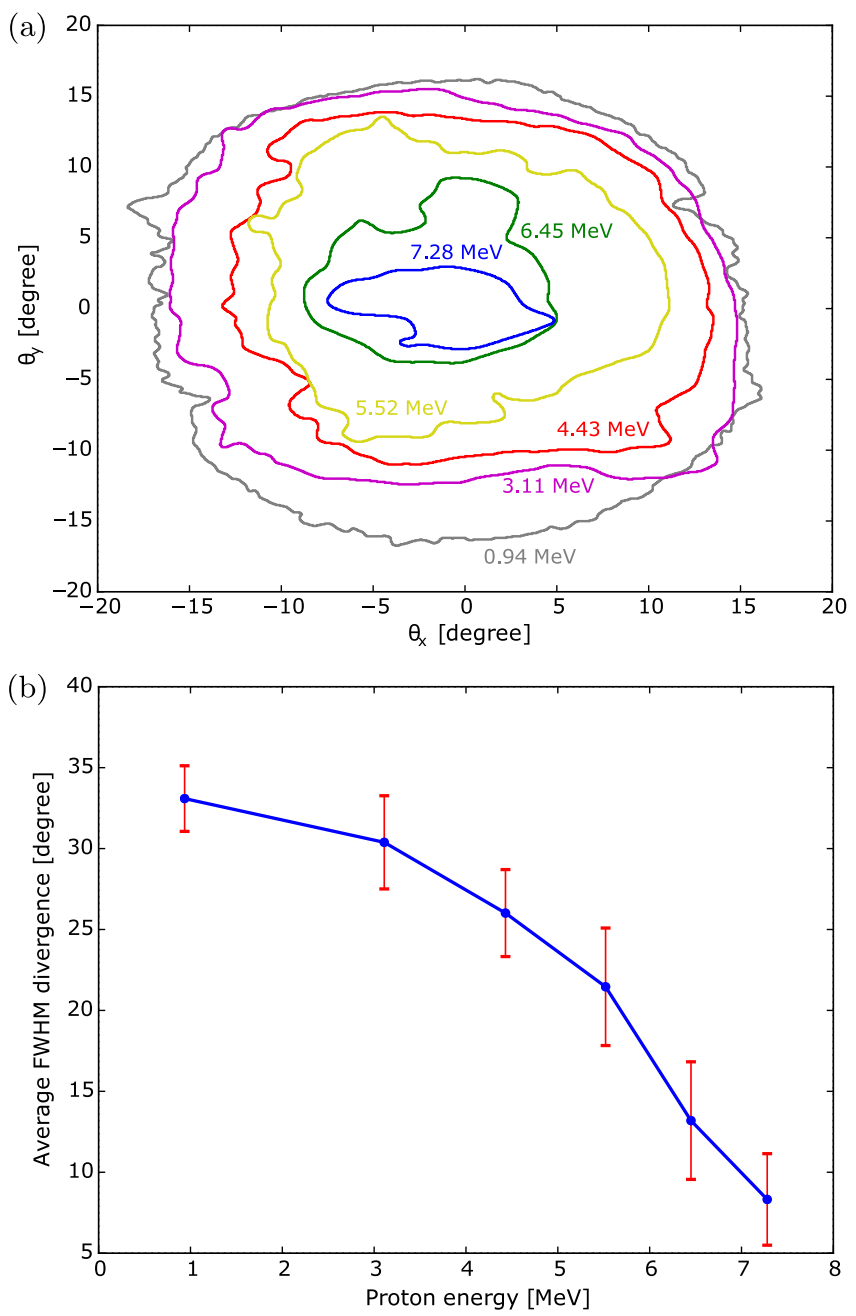

FIG. 1. (a) Energy dependent divergence of the proton beam source. The contours drawn are the isodose lines at $50 \%$ of the maximum dose, and the labels indicate the minimum energy required for a proton to reach the considered radiochromic film. (b) Average divergence of the proton beam, calculated from (a) as function of its energy.

PMQ lenses have the advantage of being relatively compact while producing a high field gradient within a relatively large aperture. In the present case, the system consists of two sets of two PMQs of 40 and $80 \mathrm{~mm}$ length, with a magnetic field gradient of about $100 \mathrm{Tm}^{-1}$ inside a $2 \mathrm{~cm}$ diameter net bore. The compact size enables us to install the device inside the interaction chamber as close as possible to the interaction point. The distance separating the PMQs can be changed to adjust the output beam according to the required size and energy.

The final configuration for the transport beam line is illustrated in Fig. 2. The first quadrupole (PMQ1) was placed $5 \mathrm{~cm}$ behind the target. Despite the high beam divergence (see Fig. 1), this enables one to collect all the protons above $4 \mathrm{MeV}$ at the entrance of PMQ1, which includes all the particles that have a sufficient energy to reach the output sample. 


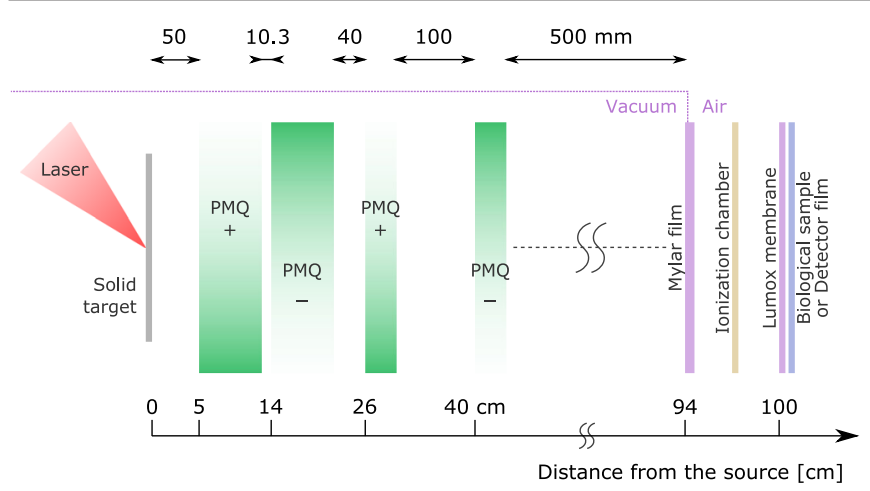

FIG. 2. Sketch of the SAPHIR beam line setup used for proton beam transport studies. The first PMQ is set at the minimal distance of $5 \mathrm{~cm}$ from the source. The plus and minus symbols indicate the relative polarity of each PMQ. The $75 \mu \mathrm{m}$ Mylar film for vacuum/air separation and the transmission ionization chamber are also depicted.

The relative spacings between the PMQ elements were optimized using the TraceWin Multiparticle calculation code. The simulated envelope, along with the particle losses for a $5 \mathrm{MeV}$ beam, are shown in Fig. 3. The aim of this configuration was to limit the losses along the transport, while obtaining a relatively large output beam with a low divergence. The goal was also to filter the beam in order to get a narrow energy spectrum with a Bragg peak located closely behind the sample layer, so as to maximize the dose deposition in the sample to be irradiated. Most of the losses occur in the first PMQ as protons are defocused along the $x$ axis, but the transmission efficiency of $15 \%$ for the selected energy of $5 \mathrm{MeV}$ is a remarkably high figure. The beam size obtained at $56 \mathrm{~cm}$ from the last PMQ is $10 \mathrm{~mm} \times 15 \mathrm{~mm}$. In perspective, one could also consider using octupoles to achieve a better beam profile uniformity $[33,34]$.

\section{B. Energy selection}

PMQs are chromatic so a given configuration is optimized for a single energy component of the beam. This is demonstrated by simulations showing large losses for protons with energies outside the range for which the setup was optimized. It is however impossible to adjust the position of the various PMQs to get the desired profiles for several proton energies independently.

The spectrum is measured at the end of the beam line, right before the Mylar foil (see Fig. 2) by a Thomson parabola spectrometer (TPS). Tracks are visualized on an microchannel plate detector to retrieve the proton beam spectrum. The effect on the final spectrum, simulated from the measured proton spectrum, is shown in Fig. 4 (the code proved to match well experimental observations [32]). As expected the transmission efficiency increases at higher energies [Fig. 4(a)]. The output proton spectrum is narrower and centered around $5 \mathrm{MeV}$ [Fig. 4(b)].

Even though some carbon and oxygen ions are detected by the TPS, no ions other than protons will be found in the
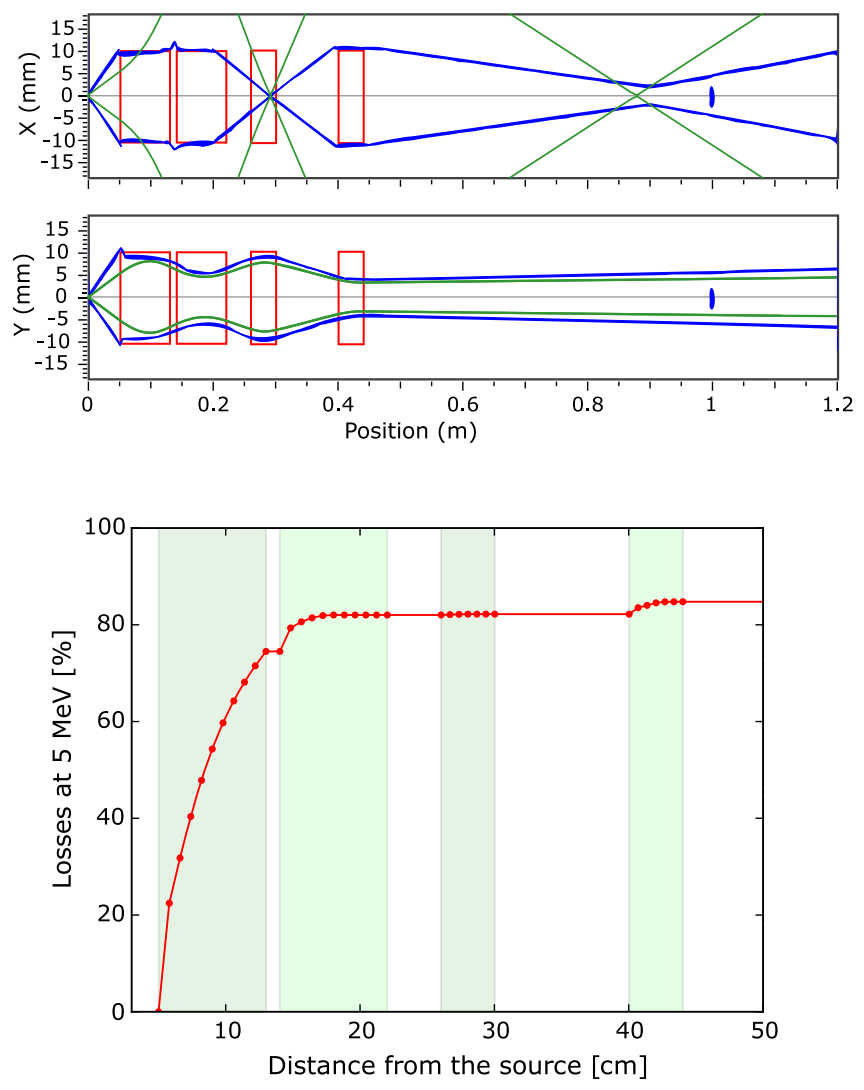

FIG. 3. $5 \mathrm{MeV}$ proton beam simulations performed with the TraceWin Multiparticle tool in the described PMQ setup. The top pictures show the calculated envelope in the $X$ and $Y$ directions, with PMQs represented by the red rectangles, the actual beam envelope in blue, and the rms aperture-free beam envelope in green. The particle losses are plotted in the bottom graph, where the shaded area represents the PMQs.

beam propagating in air as all heavy ions at the considered sub-MeV energies are stopped in the Mylar window. Concerning protons, they need to have an energy greater than $4.4 \mathrm{MeV}$ to propagate through the entire system without being stopped before depositing energy in the output sample. Relativistic electrons are also generated during the laser-plasma interaction, but they are completely rejected by the PMQ system. According to simulations, electrons with initial energies lower than $10 \mathrm{MeV}$ are not able to reach the vacuum chamber exit. An $x$-ray signal is emitted from the plasma in $4 \pi$ sr so some of it reaches the sample, which is in a direct line of sight of the source point. Its intensity was recorded on an IP in a configuration not transmitting particles and the measurement was 3 orders of magnitude lower than the proton signal obtained in regular conditions.

\section{DOSIMETRY}

In order to monitor the proton source for variations, e.g. the laser parameters as well as target positioning 


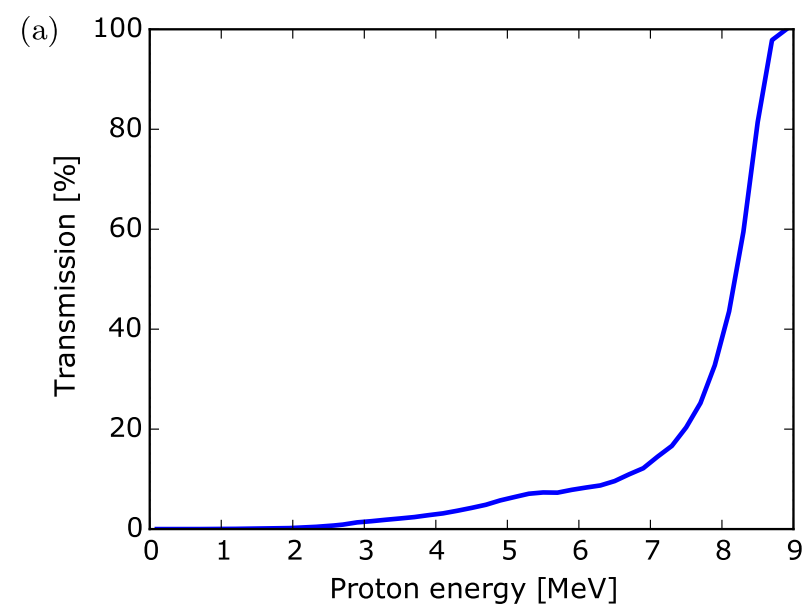

(b)

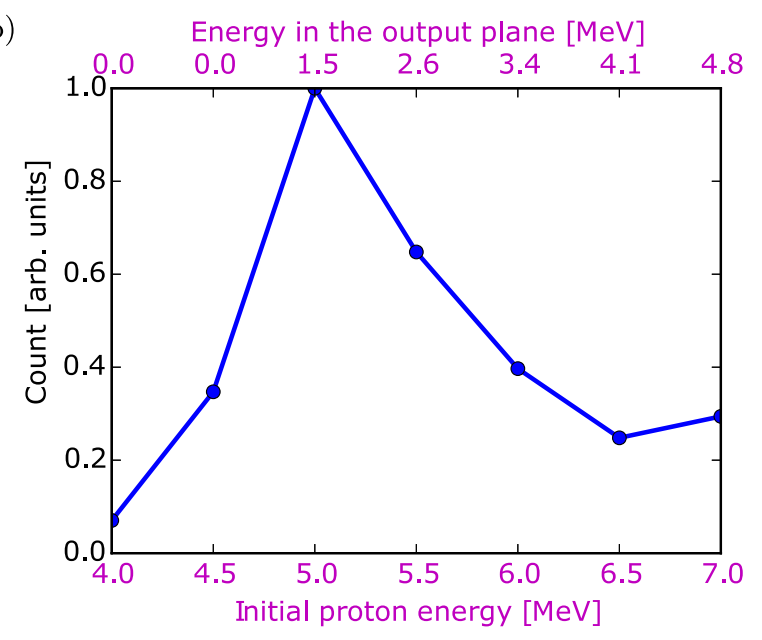

FIG. 4. (a) Transmission efficiency of the PMQ system as a function of the proton energy. (b) Relative energy spectrum of the proton beam reaching the $10 \times 15 \mathrm{~mm}^{2}$ output sample area. The bottom scale shows the initial proton energy, while the top scale shows the energy of these same particles when they reach the sample layer after crossing some absorbing elements.

and surface inhomogeneities, a transmission monitor chamber is used, allowing precise control of the final dose. A PTW transmission monitor ionization chamber model 786 was used $(155 \mathrm{~mm}$ diameter, $0.11 \mathrm{~mm}$ equivalent water thickness), combined with a PTW electrometer UNIDOS ${ }^{\circledR}$ E Universal Dosemeter to read the measurements.

The advantages of such a thin flat transmission ionization chamber (TIC) is that it can provide an online monitoring of the beam passing through it, on its way to the region of practical use. The TIC can be permanently installed while the output beam can still be used for the desired application.

\section{A. TIC calibration experiment}

The TIC has been absolutely calibrated at the Institut Curie's Proton Therapy Center in Orsay (CPO), France.

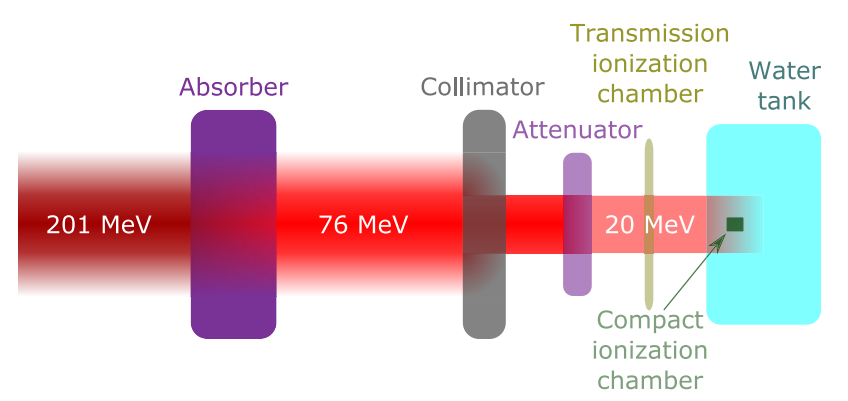

FIG. 5. Schematic of the TIC calibration setup.

The source is an IBA C230 isochronous cyclotron, which generates a proton beam with an initial energy of $235 \mathrm{MeV}$. The beam energy is lowered to $201 \mathrm{MeV}$ right at the cyclotron output and further reduced down to $76 \mathrm{MeV}$ as per treatment protocol of eye tumors. This second attenuation is achieved with $175 \mathrm{~mm}$ of polycarbonate (Lexan) and $1 \mathrm{~mm}$ of lead placed about $1 \mathrm{~m}$ after the beam exits the transport line under vacuum, and $5 \mathrm{~m}$ before the irradiation spot. A final attenuation to $20 \mathrm{MeV}$ is used for our experiment, thanks to $36.1 \mathrm{~mm}$ of Plexiglas placed in front of the TIC. The beam was also shaped with a collimator to a square aperture of side $2 \mathrm{~cm}$ (see a drawing of the setup in Fig. 5 and the energy deposition transverse profile of the CPO beam in Fig. 6).

The TIC is calibrated against the reference ionization chamber (compact thimble air ionization chamber CC13) which is routinely used at the CPO. Combining the measurements of both ionization chambers, the dose deposition in the $\mathrm{CC} 13$ is related to the charge measured in the TIC by the linear relationship:

$$
\begin{aligned}
\eta_{\text {indirect }} & =\frac{\text { Dose }_{\mathrm{CC} 13}}{Q_{\mathrm{TIC}}} \\
& =1.6 \pm 0.1 \mathrm{cGy}_{\mathrm{CC} 13} / \mathrm{nC}_{\mathrm{TIC}}
\end{aligned}
$$

where subscripts have been added to the units to remind to which element they are associated to.
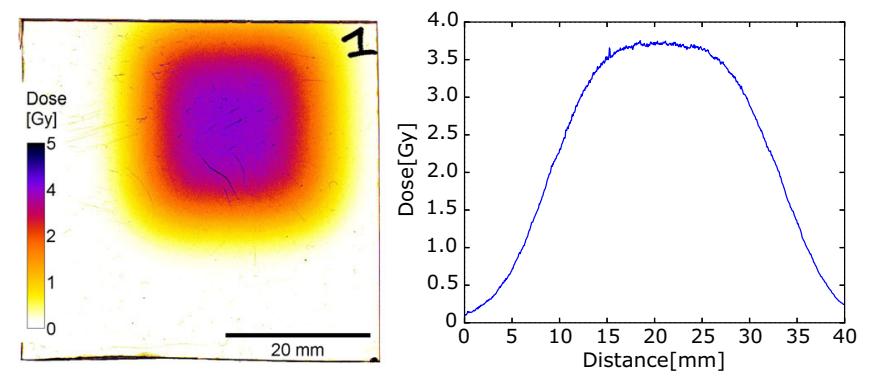

FIG. 6. Dose deposition profile of the CPO proton beam recorded on a stack of RCF EBT2 placed behind the collimator. The graph on the right shows an averaged horizontal lineout of the dose map. 


\section{B. Simulations for absolute dose retrieval}

In order to retrieve the TIC calibration for the output dose deposition at the lower proton energies provided by the SAPHIR laser-plasma accelerator, Monte Carlo simulations have been run with the Geant4 toolkit [35]. In a first step, the CPO calibration setup (Fig. 5) was simulated to get the absolute dose calibration of the TIC. The ratio of average dose deposited per primary proton in the CC13 and TIC sensitive air volumes is

$$
R_{\mathrm{dose}}^{\mathrm{CPO}}=\frac{\operatorname{Dose}_{\mathrm{TIC}}^{\mathrm{Simu}}}{\operatorname{Dose}_{\mathrm{CC} 13}^{\mathrm{Simu}}}=0.14 .
$$

The proton beam fluence was well uniform in the area imposed by the $2 \mathrm{~cm}$ side square collimator aperture, as indicated by the scan of the RCF EBT2 stack placed behind it and displayed in Fig. 6. Indeed, the FWHM of the dose deposition signal is $24.7 \mathrm{~mm}$ and the signal has a $20 \mathrm{~mm}$ width at $70 \%$ of the maximum intensity. The ratio of total deposited dose in the ionization chambers should take into account the geometrical factor of irradiated areas. In fact while the $\mathrm{CC} 13$, surface $5 \times 5 \mathrm{~mm}^{2}$, was fully illuminated, the beam cross section is considerably smaller than the TIC active surface, which limits the irradiation to $2 \times 2 \mathrm{~cm}^{2}$ of its useful diameter, as set by the square beam collimator. The illuminated section ratio is

$$
R_{\mathrm{surf}}=\frac{S_{\mathrm{TIC}}}{S_{\mathrm{CC} 13}}=\frac{4}{0.25}=16,
$$

which gives, for the comparison of the dose deposition in the two devices for the actual CPO proton beam, the dose ratio:

$$
\eta_{\text {dose }}=R_{\text {surf }} \times R_{\text {dose }}^{\mathrm{CPO}}=2.3 .
$$

Finally, combining the TIC indirect calibration in Equation (1) with the above dose ratio, the intrinsic TIC calibration represented by the factor correlating the charged measured in the TIC with the dose deposition inside its active air volume is obtained:

$$
\begin{aligned}
\eta_{\mathrm{TIC}} & =\frac{\text { Dose }_{\mathrm{TIC}}}{Q_{\mathrm{TIC}}}=\eta_{\text {dose }} \times \eta_{\text {indirect }} \\
& =3.7 \mathrm{cGy}_{\mathrm{TIC}} / \mathrm{nC}_{\mathrm{TIC}}
\end{aligned}
$$

In a second step, the absorbing elements of the SAPHIR beam line (Fig. 2) were implemented to simulate the relative energy deposition in the TIC and the cell monolayer sample. Coupling these Geant4 energy deposition simulations with the energy-dependent spatial distribution obtained by propagating our proton source beam through the system with TraceWin, a realistic spatially dependent spectrum was computed in each of the nine sections of the $1.5 \mathrm{~cm}^{2}$ sample area, which was divided into a $3 \times 3$ grid. The average spectrum over the full sample area, delimited by the white rectangle in Fig. 8, is shown in Fig. 4(b). The average energy deposition ratio between the sample area and the TIC is $E_{\text {Cell } / \mathrm{TIC}}=1.02$. Applying a $W_{\mathrm{TIC} / \text { Cell }}=3.03$ normalization factor accounting for the weight ratio of the TIC irradiated air volume at atmospheric pressure, and the cell sample regarded as water (dose is inversely proportional to mass), leads to an average dose ratio of

$$
R_{\mathrm{dose}}^{\mathrm{Saphir}}=\frac{\operatorname{Dose}_{\mathrm{Cell}}^{\mathrm{Simu}}}{\operatorname{Dose}_{\mathrm{TIC}}^{\mathrm{Simu}}}=W_{\mathrm{TIC} / \text { Cell }} \times E_{\mathrm{Cell} / \mathrm{TIC}}=3.1 .
$$

The final calibration, relating the average dose deposition in the biological sample with the charge measured in the TIC, is deduced combining Eqs. (5) and (6):

$$
\begin{aligned}
\eta_{\text {Cell }} & =\frac{\text { Dose }_{\text {Cell }}}{Q_{\text {TIC }}}=R_{\text {dose }}^{\text {Saphir }} \times \eta_{\text {TIC }} \\
& =11.5 \pm 5.9 \mathrm{cGy}_{\text {Cell }} / \mathrm{nC}_{\mathrm{TIC}} .
\end{aligned}
$$

The figures given previously are averaged on the nine individual calibration values obtained from the spatially resolved simulations performed for each grid area of the sample. The indicated $51 \%$ error is associated to the spatial variations observed over the nine sample sections, but it could have been overestimated due to some spatial beam features that are blurred after the beam propagates through the absorbing elements at the end of the beam line. Indeed, the standard deviation calculated from the signal recorded on the IP (in the white rectangle corresponding to the sample area illustrated in Fig. 8) is only $22 \%$. This latter value is retained as the calibration error.

\section{Irradiation conditions}

A proton accelerated to an initial energy of $5 \mathrm{MeV}$ (energy corresponding to the peak in the output spectrum) crosses the transmission ionization chamber (TIC) with an energy of $4 \mathrm{MeV}$ while depositing $D_{\mathrm{TIC}}^{1 H^{+}}=$ 4.0 nGy (see Fig. 7). The charge measured experimentally was around $Q_{\mathrm{TIC}}^{\exp }=10 \mathrm{nC}$ per laser shot, with a standard deviation of $\sigma=15 \%$ for a typical day with over a hundred shots, so according to the calibration (5) for our output proton beam passing through the TIC, it corresponds to a dose deposition in it of

$$
D_{\mathrm{TIC}}^{\exp }=Q_{\mathrm{TIC}}^{\exp } \times \eta_{\mathrm{TIC}}=37 \pm 5.6 \mathrm{cGy} .
$$

Hence, according to the calibration in (7), the average dose per shot received by the analyzed biological sample area is 


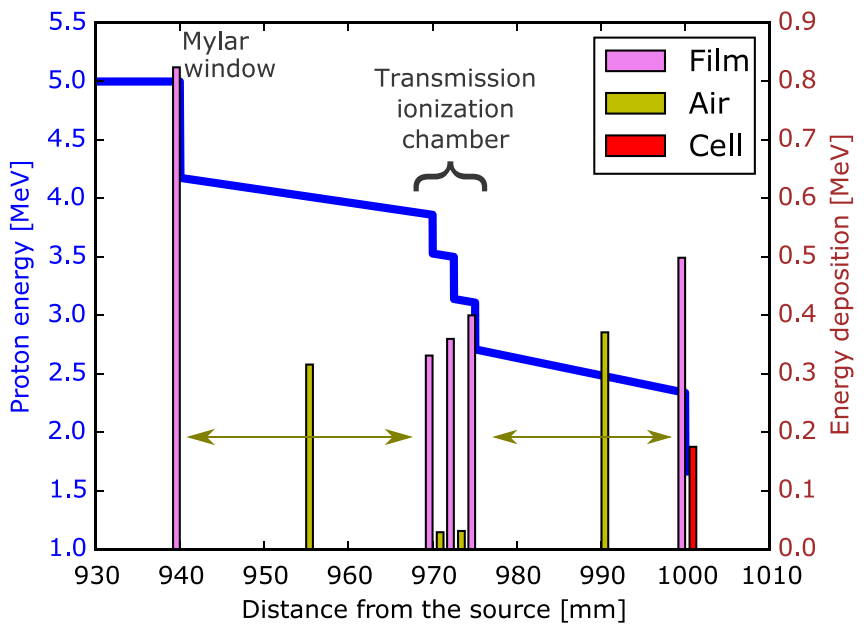

FIG. 7. Graph depicting the energy decrease (blue curve, vertical scale on the left) of an instance proton with a $5 \mathrm{MeV}$ initial energy, as it propagates along the beam line. The energy deposited in the various absorbing elements encountered on its path is represented by the bar plot (vertical scale on the right). Each bar is centered in its energy deposition longitudinal range to preserve proportionality between the energy deposition value and the area of the corresponding bar.

$$
D_{\text {Cell }}^{\exp }=\eta_{\text {Cell }} \times Q_{\text {TIC }}^{\exp }=1.15 \pm 0.25 \mathrm{~Gy} / \text { shot },
$$

and the number of output protons can be estimated to

$$
\begin{aligned}
N_{H^{+}}^{\text {Cell }} & =\frac{D_{\text {TIC }}^{\exp }}{D_{\text {TIC }}^{1 H^{+}}}=\frac{0.37}{4.0 \times 10^{-9}} \\
& =9.3 \times 10^{7} \text { protons per shot. }
\end{aligned}
$$

The corresponding average fluence in the sample area, marked out by the white rectangle in Fig. 8, is

$$
F_{\text {Cell }}=\frac{N_{H^{+}}^{\text {Cell }}}{A_{\text {Cell }}}=6.2 \times 10^{7} \text { protons } / \mathrm{cm}^{2} .
$$

The accelerated proton bunch has an emission duration at the source in the order of a few picoseconds [36]. After propagation over the one meter path length to reach the sample, the transmitted protons of the beam in the 4.5-6.5 MeV energy range are spread out in space due to their speed differential, and irradiate the sample for a duration of $\Delta t=5.7 \mathrm{~ns}$. The peak dose rate inflicted to the biological sample is estimated to be

$$
\phi_{S}=\frac{D_{\mathrm{Cell}}^{\mathrm{exp}}}{\Delta t}=2.0 \times 10^{8} \mathrm{~Gy} / \mathrm{s} .
$$

\section{RADIOBIOLOGY EXPERIMENTS}

The main goal of the present proton transport system optimization was to adapt the SAPHIR beam to biological studies. In order to get a stronger confirmation

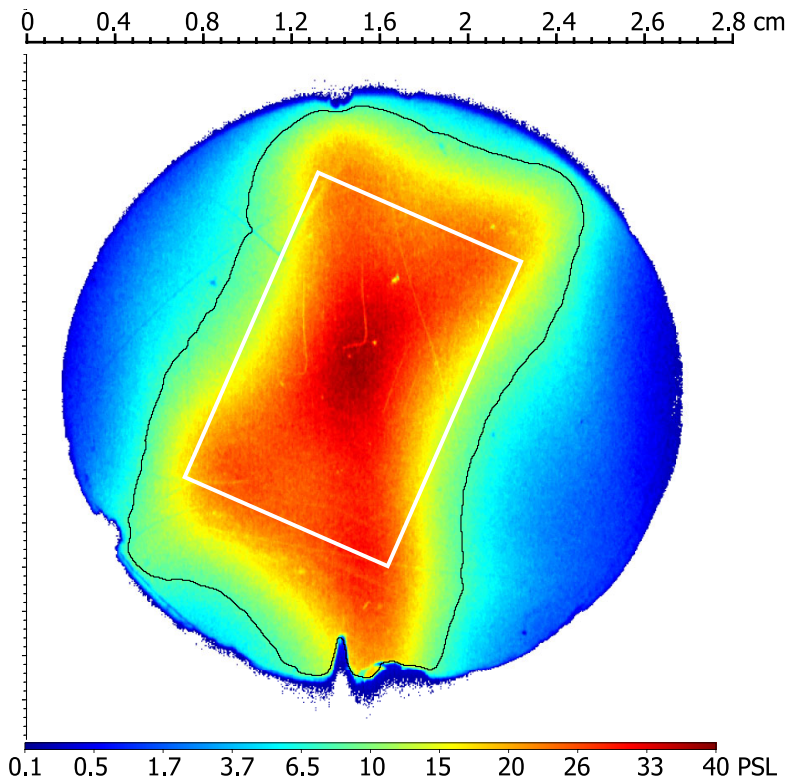

FIG. 8. Transverse profile of the proton beam dose deposition at the transport line output, recorded on an imaging plate with $28 \mathrm{~mm}$ diameter. The IP was placed $1 \mathrm{~m}$ away from the target, at the biological sample location in air. The isodose curve at 8 PSL (i.e. $20 \%$ of the maximum recorded signal) is represented by the black line, and the white rectangle marks the area where the biological sample response was studied.

on the dosimetry protocol, radiobiological effects of laser-accelerated pulsed protons were assessed on human colorectal cancer HCT116 cells.

\section{A. Foci analysis}

We first analyzed DNA damage foci resulting from SAPHIR accelerated protons. The HCT116 cells, wild type (WT) or mutated for the tumor suppressor gene p53 $\left(\mathrm{p} 53^{-/-}\right.$) and known to be more radioresistant, were cultured in McCoy's 5A (Modified) Medium (ThermoFisher Scientific), supplemented with $10 \%$ fetal calf serum and

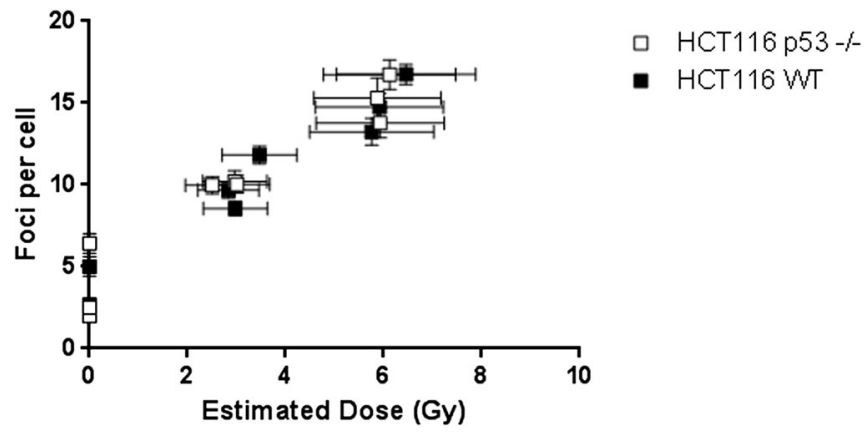

FIG. 9. Number of $\gamma \mathrm{H} 2 \mathrm{AX}$ foci generated in HCT116 WT and p53 $3^{-/-}$cells irradiated with an increasing dose provided by four or eight shots of laser-accelerated protons $(n=3)$. For each data point, foci were determined from at least 200 nuclei. 
$1 \%$ penicillin and streptomycin. Cells were grown at $37^{\circ} \mathrm{C}$ in a humidified atmosphere of $5 \% \mathrm{CO}_{2}$ in air. The cell containers used for irradiation were lumox ${ }^{\circledR}$ dish 35 (SARSTEDT) exhibiting a $25 \mu \mathrm{m}$ thick lumox ${ }^{\circledR}$ bottom face. $4 \times 10^{5}$ cells were seeded on the entire lumox surface and were grown overnight. The culture dish was positioned vertically behind the exit window, outside of the vacuum chamber and behind the ionization chamber. Cells were subjected to four or eight successive shots. Appropriate control samples were treated under the same conditions

(a)
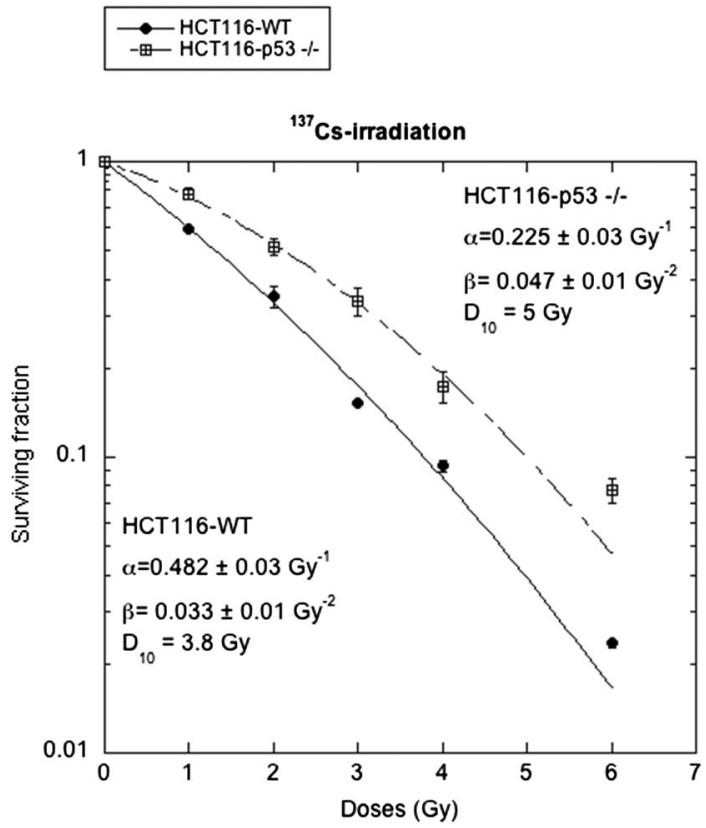

(c)

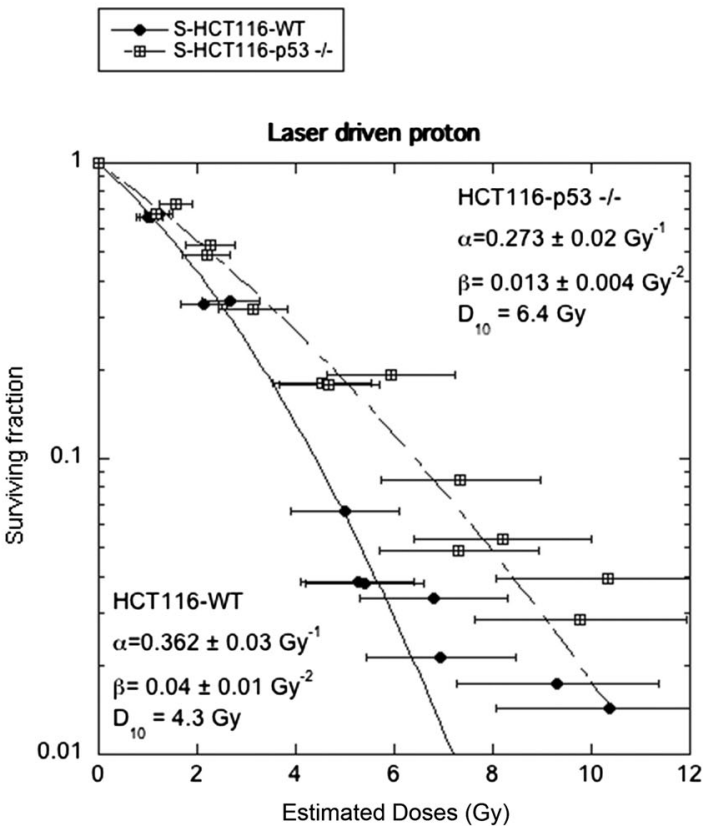

including bringing the cell culture dish in a vertical position as for irradiation.

The cells were incubated one hour post irradiation at $37^{\circ} \mathrm{C}$ and then fixed in $4 \%$ Formalin solution (Sigma). After cell permeabilization $(0.5 \%$ Triton X-100) and saturation (2\% FSC), the irradiation surface was delimitated with Dako Pen (Agilent). The cells were incubated with a mouse monoclonal antibody against phosphorylated form of the variant histone $\mathrm{H} 2 \mathrm{AX}(\gamma \mathrm{H} 2 \mathrm{AX}$, Merck) and then with a secondary antibody (Alexafluor 546 goat

(b)

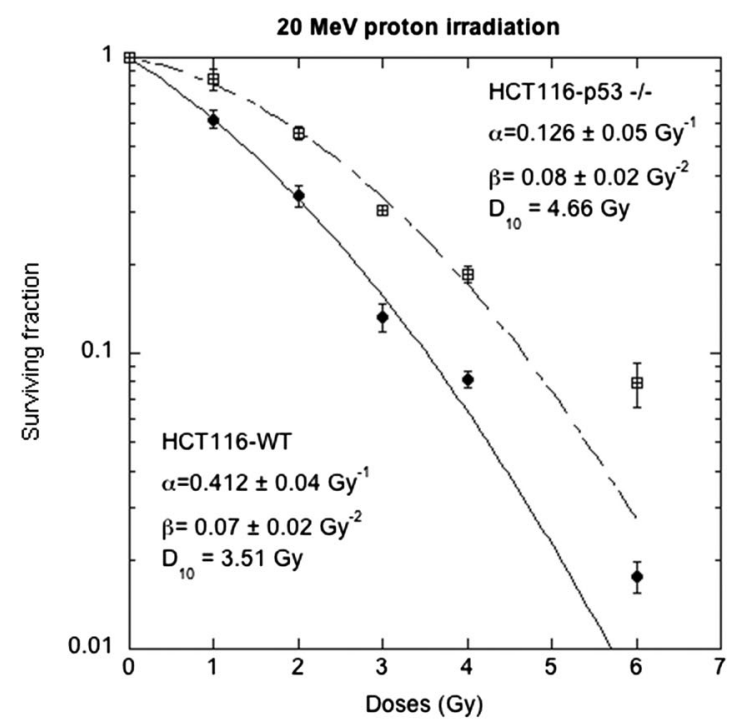

(d)

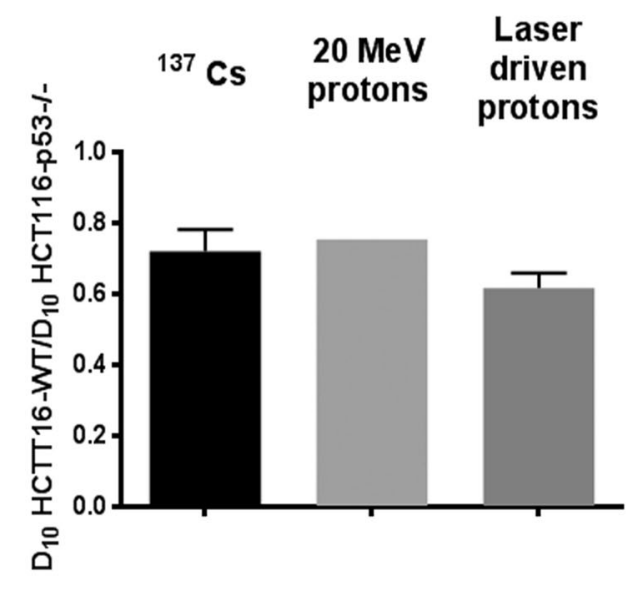

FIG. 10. Survival curves of HCT116-WT and $\mathrm{p} 53^{-/-}$cells after ${ }^{137} \mathrm{Cs}$ (a, mean $\pm \mathrm{SD}$ of three independent experiments), $20 \mathrm{MeV}$ proton ( $b$, mean \pm SD of two independent experiments) or laser-driven irradiations (c, mean \pm SD of three independent experiments). (d) Calculated D10 ratio between HCT116-WT and $\mathrm{p} 53^{-/-}$cells for each irradiation condition. 
anti-mouse antibody, ThermoFisher Scientific). Picture acquisition was performed using DMi8 microscope and LASX software (Leica) at 40x magnification. Foci were automatically counted in each nucleus, using a home-made ImageJ macro and represented as a function of the applied dose. As shown in Fig. 9, increasing doses led to increasing amounts of detected foci in cell nuclei. For each irradiation condition, similar amounts of foci were counted in the two cell lines. These results suggest that despite the theoretical importance of the error on the dose, the DNA damage generated by laser pulsed protons showed a great reproducibility of the dose delivered to the sample.

\section{B. Survival assays}

In a second step, survival assays were performed. Depending on beam form and position deducted from image plate acquisition, a $9 \mathrm{~mm} \times 18 \mathrm{~mm}$ area was delimited in which $6 \times 10^{4}$ cells were seeded. After exposure to laser-driven protons, cells were incubated for 4 hours and harvested with Accutase (Merck). Cells were then dispatched into three different wells of 12-well plate and placed in the incubator. Survival assays were also performed with the $20 \mathrm{MeV}$ proton beam used at the Institut Curie Proton Therapy Center [37,38] and the $662 \mathrm{keV}$ emission of a ${ }^{137} \mathrm{Cs}$ source. Cells were seeded at a density of $4 \times 10^{4}$ cells $/ \mathrm{cm}$ in $25 \mathrm{~cm}^{2}$ flasks, then exposed to $20 \mathrm{MeV}$ protons $\left(3 \mathrm{~Gy} \mathrm{~min}^{-1}\right.$ ) or ${ }^{137} \mathrm{Cs} \gamma$-rays $\left(1 \mathrm{~Gy} \mathrm{~min}^{-1}\right.$ ) and returned to the incubator for 4 hours. The cells were then harvested and replated in six-well plates in triplicate at a density of $4 \times 10^{3}$ cells $/ \mathrm{cm}$. After five days, cells were harvested and counted using flow cytometry technology (Guava $^{\circledR}$, Millipore) and the exclusion of a propidium iodide solution added just before analysis (GuavaSoft).

Cell survival $(S)$ curves were fitted to the linearquadratic equation: $\ln S=\alpha D-\beta D^{2}$, where $D$ is the dose of radiation and $\alpha$ and $\beta$ are adjustable parameters. For each experiment the $10 \%$ survival dose $\left(D_{10}\right)$ was calculated using the $\alpha$ and $\beta$ parameters determined from curves drawn for best fit to the experimental data (Kaleidagraph software). Cell survival values obtained in response to increasing doses of irradiation with laser accelerated protons allowed one to perform a reliable linear quadratic regression analysis [Fig. 10(c)], as for ${ }^{137}$ cesium or $20 \mathrm{MeV}$ conventional protons [Fig. 10(a) and 10(b)]. As shown in Fig. $10(\mathrm{~d})$, the $D_{10}$ ratio between HCT116-WT and HCT116-p53-/- corresponding to pulsed protons $(0.62 \pm 0.4)$ appears to be reduced compared to those of $20 \mathrm{MeV}$ conventional protons and $662 \mathrm{keV}$ photons $(0.75$ and $0.72 \pm 0.06$ respectively). However, this apparent reduction in effectiveness of the pulsed protons was not statistically significant $(P>0.05)$. It results probably from the relatively large error but was acceptable for the proof of principle experiments for use of the SAPHIR facility for radiobiological studies. All together, these results reflected the good control of the dosimetry to sample and the stability of the beam. The uncertainty on the dose measurement in this study is comparable to others [18,39-41], although the useful irradiation surface in our case is much wider.

\section{CONCLUSIONS}

In conclusion, we designed and implemented an efficient transport line for TNSA accelerated protons. Out of a divergent emission with exponential spectrum to $8 \mathrm{MeV}$, a limited spectrum, $5 \mathrm{MeV}$ proton beam was produced. The transport line was optimized to produce a uniform spot of $15 \mathrm{~mm} \times 25 \mathrm{~mm}$ in air at a distance of $1 \mathrm{~m}$ from the source. The $15 \%$ transport efficiency of the energy component of interest can be improved using a solenoid $[12,42]$. Its radial magnetic field ensures a more efficient initial collection of the proton beam.

Online dosimetry, provided by a calibrated transmission ionization chamber, showed a transported charge of $9 \times 10^{7}$ protons per shot. Dosimetry is confirmed by survival curves of HCT116. The dose deposited in the cell monolayer culture is $1.15 \pm 0.25 \mathrm{~Gy} / \mathrm{shot}$, with an estimated peak dose rate exceeding $10^{8} \mathrm{~Gy} / \mathrm{s}$. These results open the way to extensive study of the effects of ultrahigh dose deposition rates in living matter.

Laser-accelerated ions are still far from clinical use [43]. For instance, accelerating with laser pulses protons up to $200 \mathrm{MeV}$, the energies required for protontherapy, remains a major challenge that is currently motivating numerous research studies in the scientific community. The present work offers a system that can be used to perform radiobiological studies in the ten $\mathrm{MeV}$ proton range that is achieved nowadays by several laser research facilities worldwide. This is a relevant energy for biological studies as a proton beam with an initial energy of a couple hundreds of $\mathrm{MeV}$ will be attenuated down to such an energy level when it reaches a target tumor located at a depth inside a body. The most important cellular damage is occurring at low proton energy, with the relative biological effectiveness peaking for a linear energy transfer of about $100 \mathrm{keV} \mu \mathrm{m}^{1}$, which corresponds to $0.1 \mathrm{MeV}$ protons. As the proton energy is scaled up, stronger focusing fields will have to be used and the transport line technology will have to be adapted. Designs have already been envisioned based on pulsed magnet optics [44].

\section{ACKNOWLEDGMENTS}

The authors acknowledge the support of OSEO Project No. I0901001W-SAPHIR and of the European Research Council through the X-Five ERC project (Contract No. 339128). E. B. acknowledges support from the European Union's Horizon 2020 research and innovation program under Grant Agreement No. 654148 LaserlabEurope. 
[1] I. J. Kim, K. H. Pae, C. M. Kim, H. T. Kim, J. H. Sung, S. K. Lee, T. J. Yu, I. W. Choi, C.-L. Lee, K. H. Nam et al., Transition of Proton Energy Scaling Using an Ultrathin Target Irradiated by Linearly Polarized Femtosecond Laser Pulses, Phys. Rev. Lett. 111, 165003 (2013).

[2] T. Esirkepov, M. Borghesi, S. V. Bulanov, G. Mourou, and T. Tajima, Highly Efficient Relativistic-Ion Generation in the Laser-Piston Regime, Phys. Rev. Lett. 92, 175003 (2004).

[3] L. Yin, B. J. Albright, B. M. Hegelich, K. J. Bowers, K. A. Flippo, T. J. T. Kwan, and J. C. Fernandez, Monoenergetic and $\mathrm{GeV}$ ion acceleration from the laser breakout afterburner using ultrathin targets, Phys. Plasmas 14, 056706 (2007).

[4] D. Haberberger, S. Tochitsky, F. Fiuza, C. Gong, R. A. Fonseca, L. O. Silva, W. B. Mori, and C. Joshi, Collisionless shocks in laser-produced plasma generate monoenergetic high-energy proton beams, Nat. Phys. 8, 95 (2012).

[5] A. Yogo, K. Sato, M. Nishikino, M. Mori, T. Teshima, H. Numasaki, M. Murakami, Y. Demizu, S. Akagi, S. Nagayama et al., Application of laser-accelerated protons to the demonstration of DNA double-strand breaks in human cancer cells, Appl. Phys. Lett. 94, 181502 (2009).

[6] A. Macchi, M. Borghesi, and M. Passoni, Ion acceleration by superintense laser-plasma interaction, Rev. Mod. Phys. 85, 751 (2013).

[7] M. Schollmeier, S. Becker, M. Geiel, K. A. Flippo, A. Blaevi, S. A. Gaillard, D. C. Gautier, F. Grner, K. Harres, M. Kimmel et al., Controlled Transport and Focusing of Laser-Accelerated Protons with Miniature Magnetic Devices, Phys. Rev. Lett. 101, 055004 (2008).

[8] M. Nishiuchi, I. Daito, M. Ikegami, H. Daido, M. Mori, S. Orimo, K. Ogura, A. Sagisaka, A. Yogo, A. S. Pirozhkov et al., Focusing and spectral enhancement of a repetitionrated, laser-driven, divergent multi-MeV proton beam using permanent quadrupole magnets, Appl. Phys. Lett. 94, 061107 (2009).

[9] F. O'Shea, G. Andonian, and J. B. Rosenzweig, Permanent magnet quadrupole final focus system for the muon collider, Conf. Proc. C100523, 3524 (2010).

[10] V. Kumar, Understanding the focusing of charged particle beams in a solenoid magnetic field, Am. J. Phys. 77, 737 (2009).

[11] K. Harres, I. Alber, A. Tauschwitz, V. Bagnoud, H. Daido, M. Gnther, F. Nrnberg, A. Otten, M. Schollmeier, J. Schtrumpf et al., Beam collimation and transport of quasineutral laser-accelerated protons by a solenoid field, Phys. Plasmas 17, 023107 (2010).

[12] T. Burris-Mog, K. Harres, F. Nrnberg, S. Busold, M. Bussmann, O. Deppert, G. Hoffmeister, M. Joost, M. Sobiella, A. Tauschwitz et al., Laser accelerated protons captured and transported by a pulse power solenoid, Phys. Rev. ST Accel. Beams 14, 121301 (2011).

[13] Y. Oishi, D. Giulietti, F. Baffigi, L. Fulgentini, A. Giulietti, P. Kster, L. Labate, M. Kando, and L. A. Gizzi, Focusing and stabilizing laser-plasma-generated electron beams with magnetic devices, Jpn. J. Appl. Phys. 53, 092702 (2014).

[14] H. Schwoerer, S. Pfotenhauer, O. Jäckel, K.-U. Amthor, B. Liesfeld, W. Ziegler, R. Sauerbrey, K. W. D. Ledingham, and T. Esirkepov, Laser-plasma acceleration of quasi-monoenergetic protons from microstructured targets, Nature (London) 439, 445 (2006).

[15] J. Weichsel, T. Fuchs, E. Lefebvre, E. d'Humires, and U. Oelfke, Spectral features of laser-accelerated protons for radiotherapy applications, Phys. Medicine Biol. 53, 4383 (2008).

[16] E. Fourkal, J. S. Li, M. Ding, T. Tajima, and C.-M. Ma, Particle selection for laser-accelerated proton therapy feasibility study, Med. Phys. 30, 1660 (2003).

[17] A. Yogo, T. Maeda, T. Hori, H. Sakaki, K. Ogura, M. Nishiuchi, A. Sagisaka, P. R. Bolton, M. Murakami, S. Kawanishi et al., Development of laser-driven quasimonoenergetic proton beam line for radiobiology, Nucl. Instrum. Methods Phys. Res., Sect. A 653, 189 (2011).

[18] J. Bin, K. Allinger, W. Assmann, G. Dollinger, G. A. Drexler, A. A. Friedl, D. Habs, P. Hilz, R. Hoerlein, N. Humble et al., A laser-driven nanosecond proton source for radiobiological studies, Appl. Phys. Lett. 101, 243701 (2012).

[19] S. D. Kraft, C. Richter, K. Zeil, M. Baumann, E. Beyreuther, S. Bock, M. Bussmann, T. E. Cowan, Y. Dammene, W. Enghardt et al., Dose-dependent biological damage of tumour cells by laser-accelerated proton beams, New J. Phys. 12, 085003 (2010).

[20] U. Linz and J. Alonso, What will it take for laser driven proton accelerators to be applied to tumor therapy?, Phys. Rev. ST Accel. Beams 10, 094801 (2007).

[21] V. Malka, J. Faure, Y. A. Gauduel, E. Lefebvre, A. Rousse, and K. T. Phuoc, Principles and applications of compact laser-plasma accelerators, Nat. Phys. 4, 447 (2008).

[22] K. W. D. Ledingham, P. R. Bolton, N. Shikazono, and C.-M. C. Ma, Towards laser driven hadron cancer radiotherapy: A review of progress, Appl. Sci. 4, 402 (2014).

[23] F. Fiorini, D. Kirby, M. Borghesi, D. Doria, J. C. G. Jeynes, K. F. Kakolee, S. Kar, S. K. Litt, K. J. Kirkby, M. J. Merchant et al., Dosimetry and spectral analysis of a radiobiological experiment using laser-driven proton beams, Phys. Medicine Biol. 56, 6969 (2011).

[24] E. Beyreuther, W. Enghardt, M. Kaluza, L. Karsch, L. Laschinsky, E. Lessmann, M. Nicolai, J. Pawelke, C. Richter, R. Sauerbrey et al., Establishment of technical prerequisites for cell irradiation experiments with laseraccelerated electrons, Med. Phys. 37, 1392 (2010).

[25] L. Laschinsky, M. Baumann, E. Beyreuther, W. Enghardt, M. Kaluza, L. Karsch, E. Lessmann, D. Naumburger, M. Nicolai, C. Richter et al., Radiobiological effectiveness of laser accelerated electrons in comparison to electron beams from a conventional linear accelerator, J Radiat. Res. 53, 395 (2012).

[26] C. Richter, L. Karsch, Y. Dammene, S. D. Kraft, J. Metzkes, U. Schramm, M. Schrer, M. Sobiella, A. Weber, K. Zeil et al., A dosimetric system for quantitative cell irradiation experiments with laser-accelerated protons, Phys. Medicine Biol. 56, 1529 (2011).

[27] A. Flacco, F. Sylla, M. Veltcheva, M. Carrié, R. Nuter, E. Lefebvre, D. Batani, and V. Malka, Dependence on pulse duration and foil thickness in high-contrast-laser proton acceleration, Phys. Rev. E 81, 036405 (2010).

[28] S. C. Wilks, A. B. Langdon, T. E. Cowan, M. Roth, M. Singh, S. Hatchett, M. H. Key, D. Pennington, 
A. MacKinnon, and R. A. Snavely, Energetic proton generation in ultra-intense laser-solid interactions, Phys. Plasmas 8, 542 (2001).

[29] P. Mora, Thin-foil expansion into a vacuum, Phys. Rev. E 72, 056401 (2005).

[30] F. Schillaci, G. A. P. Cirrone, G. Cuttone, M. Maggiore, L. Andó, A. Amato, M. Costa, G. Gallo, G. Korn, G. Larosa et al., Design of the ELIMAIA ion collection system, J. Instrum. 10, T12001 (2015).

[31] F. Schillaci, M. Maggiore, D. Rifuggiato, G. A. P. Cirrone, G. Cuttone, and D. Giove, Errors and optics study of a permanent magnet quadrupole system, J. Instrum. 10, T05001 (2015).

[32] F. Schillaci, L. Pommarel, F. Romano, G. Cuttone, M. Costa, D. Giove, M. Maggiore, A. D. Russo, V. Scuderi, V. Malka et al., Characterization of the ELIMED Permanent Magnets Quadrupole system prototype with laser-driven proton beams, J. Instrum. 11, T07005 (2016).

[33] P. F. Meads, A nonlinear lens system to smooth the intensity distribution of a gaussian beam, IEEE Trans. Nucl. Sci. 30, 2838 (1983).

[34] N. Tsoupas, R. Lankshear, C. J. Snead, T. E. Ward, M. Zucker, and H. A. Enge, Uniform beam distributions using octupoles, in Conference Record of the 1991 IEEE Particle Accelerator Conference (IEEE, San Francisco, California, 1991), p. 1695.

[35] S. Agostinelli, J. Allison, K. Amako, J. Apostolakis, H. Araujo, P. Arce, M. Asai, D. Axen, S. Banerjee, G. Barrand et al., Geant4-a simulation toolkit, Nucl. Instrum. Methods Phys. Res., Sect. A 506, 250 (2003).

[36] B. Dromey, M. Coughlan, L. Senje, M. Taylor, S. Kuschel, B. Villagomez-Bernabe, R. Stefanuik, G. Nersisyan, L. Stella, J. Kohanoff et al., Picosecond metrology of laserdriven proton bursts, Nat. Commun. 7, 10642 (2016).

[37] V. Calugaru, C. Nauraye, G. Noël, N. Giocanti, V. Favaudon, and F. Mégnin-Chanet, Radiobiological characterization of two therapeutic proton beams with different initial energy spectra used at the institut curie proton therapy center in orsay, Int. J. Radiat. Oncol. Biol. Phys. 81, 1136 (2011).

[38] V. Calugaru, C. Nauraye, F. P. Cordelières, D. Biard, L. D. Marzi, J. Hall, V. Favaudon, and F. Mégnin-Chanet, Involvement of the artemis protein in the relative biological efficiency observed with the $76-\mathrm{MeV}$ proton beam used at the institut curie proton therapy center in orsay, Int. J. Radiat. Oncol. Biol. Phys. 90, 36 (2014).

[39] A. Yogo, T. Maeda, T. Hori, H. Sakaki, K. Ogura, M. Nishiuchi, A. Sagisaka, H. Kiriyama, H. Okada, S. Kanazawa et al., Measurement of relative biological effectiveness of protons in human cancer cells using a laser-driven quasimonoenergetic proton beamline, Appl. Phys. Lett. 98, 053701 (2011).

[40] D. Doria, K. F. Kakolee, S. Kar, S. K. Litt, F. Fiorini, H. Ahmed, S. Green, J. C. G. Jeynes, J. Kavanagh, D. Kirby et al., Biological effectiveness on live cells of laser driven protons at dose rates exceeding $10^{9} \mathrm{~Gy} / \mathrm{s}$, AIP Adv. 2, 011209 (2012).

[41] K. Zeil, M. Baumann, E. Beyreuther, T. Burris-Mog, T. E. Cowan, W. Enghardt, L. Karsch, S. D. Kraft, L. Laschinsky, J. Metzkes et al., Dose-controlled irradiation of cancer cells with laser-accelerated proton pulses, Appl. Phys. B 110, 437 (2013).

[42] I. Hofmann, Performance of solenoids versus quadrupoles in focusing and energy selection of laser accelerated protons, Phys. Rev. ST Accel. Beams 16, 041302 (2013).

[43] U. Linz and J. Alonso, Laser-driven ion accelerators for tumor therapy revisited, Phys. Rev. Accel. Beams 19, 124802 (2016).

[44] U. Masood, M. Bussmann, T. E. Cowan, W. Enghardt, L. Karsch, F. Kroll, U. Schramm, and J. Pawelke, A compact solution for ion beam therapy with laser accelerated protons, Appl. Phys. B 117, 41 (2014). 